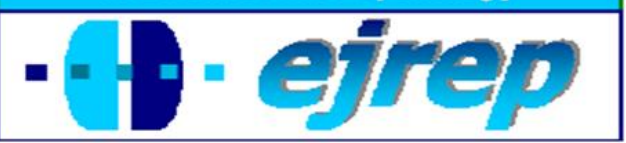

\title{
La estrategia didáctica vivencial aplicada en la enseñanza-aprendizaje de la salud en el trabajo
}

\author{
Luis Fidel Abregú Tueros ${ }^{1}$, José Luis Galve Manzano ${ }^{2}$ \\ ${ }^{1}$ Área Académica de Desarrollo Humano, Universidad Nacional Agraria de la \\ Selva, Tingo María \\ ${ }^{2}$ Colectivo para la Investigación y Desarrollos Educativos Aplicados (CIDEAS), \\ Guadalajara
}

\section{Perú / España}

Correspondencia: José Luis Galve Manzano. C/Cuesta de Calderón 2C-1B 19001, Guadalajara, España. Email: cideas@telefonica.net 


\section{Resumen}

Introducción. El estudio trata de comprobar el efecto de la estrategia didáctica de estimulación vivencial en la asimilación de contenidos y la reconstrucción de rasgos icónicos (hechos, conceptos y procedimientos) sobre la salud en el trabajo (S-T); basado en la perspectiva cognitiva fenomenográfica y del aprendizaje psicofisiológico de anclajes y reencuadres.

Método. Participaron 125 estudiantes de la Universidad Nacional Agraria de la Selva, Perú (edad: 18-20 años, 49\% varones, 51\% mujeres), 100 pertenecían al grupo de intervención (4 niveles) y 25 al grupo de referencia (GR). El diseño experimental fue totalmente al azar de una variable independiente con pre y pos prueba. Para evaluar la variable asimilación de contenidos sobre S-T, se utilizaron el test de rendimiento académico ARIST compuesto por ocho factores (80 preguntas con cuatro alternativas de respuesta cada uno).

Resultados. Según el análisis de varianza y de contingencias, las variables estudiadas presentan diferencias significativas a favor de tres niveles de tratamiento del grupo de intervención respecto al GR. Incorporándose sistemáticamente las técnicas de estimulación vivencial se logran potenciar resultados.

Discusión y conclusiones. Se evidencian la importancia de socializar las técnicas vivenciales de estimulación psíquica, que promueven el pensamiento semiótico como hábito de estudio; mejorar la calidad de la educación superior, fortaleciendo las competencias para el autoaprendizaje como las habilidades para "aprender a aprender"; y consecuentemente, elevar el desempeño en la práctica profesional de la S-T, y lograr mayor implicación entre profesores/as y estudiantes.

Palabras Clave: Enseñanza universitaria, asimilación de contenidos, memoria gráfica y de trabajo, educación para la salud, estimulación psíquica.

Recibido: 27/11/09 Aceptación Inicial: 05/12/09 Aceptación Definitiva: 27/08/10 


\title{
Experiential stimulation applied as a teaching-learning strategy for on-the-job health
}

\begin{abstract}
Introduction. The study tries to verify the effect of the didactic strategy of experiential stimulation in the assimilation of contents and the reconstruction iconic features (facts, concepts and procedures) on the health in the work (HW), based on the cognitive perspective phenomenongraphicand of the psychophysiologic learning of anchorages and resettings.

Method. 125 university students from the National Agrarian University of the Forest (Peru) participated (age 18-20 years old, $49 \%$ males, $51 \%$ females), 100 belonged to the group of intervention (four levels) and 25 to the group of reference (GR). The experimental design was totally random of an independent variable with pre and post test. To evaluate the assimilationn variable of contents on HW, they used the test of academic performance ARIST composed by eight factors ( 80 questions with four alternatives of responces each one).
\end{abstract}

Results. According to the analysis of variance and of contingencies, the studied variables present significant differences in favour of the levels of treatment of the group of intervention with regard to the GR. Joining systematicly the technologies of existential stimulation they are achieved to strengthen results.

Discussion and conclusions: There is demonstrated the importance of socializing the existential technologies of phychic stimulation that promote the semiotic thoughts as habit of study; to improve the superior education, strengthening the competences for the self learning as the skills "to learn to learn", and consistently to raise the performance in the professional practice of the HW, and to achieve major implication between teachers and students.

Keywords: University didactics, assimilation of contents, graphical memory and the work, education for health, phychic stimulation.

Received: 11/27/09 Initial Acceptance: 12/05/09 Definitive Acceptance: 08/27/10 


\section{Introducción}

La estimulación vivencial como estrategia didáctica para la enseñanza-aprendizaje en estudiantes del nivel universitario -futuros agentes mediadores de la educación para la saludespecialmente relacionada con el diseño e intervención dentro y fuera del aula, es de escasa atención, aún siendo la educación para la salud en el trabajo, el componente principal del elemento básico del desarrollo sostenible y las metodologías y acciones de promoción corresponden a las universidades. Mientras tanto, la exigencia en el diseño e intervención, como señalaran Lippke y Ziegelmann (2008), es generar cambios de comportamiento en la salud derivadas de técnicas experimentales, de tal forma, que puedan probarse empíricamente las variables predictoras de cambio en la prevención de la salud ocupacional.

Considerando que la estrategia didáctica es una acción y decisión de cómo se enseña, de por qué y para qué se enseña; que busca respuestas a los contenidos seleccionando, organizando y evaluando procedimientos coherentes entre dichos contenidos y las actividades, los medios y materiales (Cabellos et al., 2007); y facilita la enseñanza-aprendizaje (EA) y los procesos de comunicación (Sánchez, 2007). Nuestros saberes y prácticas, como objetos de reconstrucción tienen la necesidad de ser facilitadas hacia el aprendizaje autónomo de los estudiantes, y a través de un conjunto de principios de actuación comunes, desarrollar estrategias didácticas y, lograr una mayor implicancia entre los profesores y estudiantes (Díaz,1997).

Dada la resistencia natural de los estudiantes hacia los cambios de estrategias tradicionales de asimilación de contenidos en el aula, existe la necesidad de diseñar estrategias didácticas de estimulación vivencial en aula, orientándose su aplicación en los contenidos de la salud en el trabajo (S-T), que tiene como finalidad elevar el desempeño académico modificando los hábitos de estudio convencionales con las de la estimulación psíquica; bajo la premisa, de que el estudiante "solamente con el resultado de su propia experiencia vivencial, modificará su comportamiento en la asimilación de conocimientos" (Abregú, 2007, p.8). Y como tal, la estrategia didáctica vivencial circunscrita en la EA activa, tendría efectividad aún en grupos grandes y heterogénos (Cardozo et al., 2008). Además, la estrategia de intervención mediante la reconstrucción gráfica, es un proceso distintivo de lo tradicional, caracterizado por el contexto peculiar, que conforman los participantes y sus metas, así como del pensamiento simbólico, que contribuye a la reconstrucción de contenidos desde un enfoque crítico (Lacasa, Mudarra, Del Castillo, García y Martínez, 2002). 
En consecuencia, mejorando la socialización de la S-T, tomando como punto de apoyo la reconstrucción gráfica de contenidos, se favorece la conciencia crítica y la comprensión del estilo de vida y el pensamiento creativo (Lacasa et al., 2002); que podrá contribuirse hacia la calidad de la educación superior, planteados por ejemplo, en el Espacio Europeo de Educación Superior (EEES), entre otros aspectos, la promoción especial de "aprender a aprender" de los universitarios ofreciéndoles la posibilidad de ser expertos a lo largo de su vida académica y laboral (García, 2005).

La aplicación de las estrategias didácticas y su relación con el proceso de EA de estudiantes del nivel superior, son abordadas desde diversos enfoques teóricos, que dividiremos en dos grupos: cognitivo fenomenográfico y estratégico y, del aprendizaje psicofisiológico y su relación con los procesos psíquicos.

Enfoques cognitivo fenomenográfico y estratégico de la investigación sobre la enseñanzaaprendizaje en la educación superior

Desde la perspectiva cognitiva fenomenográfica planteada por Biggs (2001), referida al modo en que los estudiantes se enfrentan a la tarea, al contexto y al aprendizaje; y de la perspectiva estratégica, se concibe "como un compuesto de motivación y estrategias de aprendizaje" de tres niveles: a) superficial, que "utiliza como estrategias la memorización y la reproducción mecánica”. b) profunda, que adopta estrategias conducentes al significado de las tareas. c) de logro, que usa "como estrategia la optimización coste beneficio" (citado en García, 2005, p.49).

Al respecto, Escoriza (2005), plantea promover las competencias del alumnado basadas en el auto aprendizaje, siendo la meta, lograr el aprendizaje significativo, como resultado de la construcción del conocimiento, es decir, “aprenden a aprender", relacionando secuencialmente, las siguientes acciones: subrayar, resumir, jerarquizar ideas en función de su naturaleza inclusora; asociar usando el mapa mental, los conocimientos previos con los contenidos y con los componentes de acciones cognitivas jerarquizadas (Escoriza, 2003); que implican entre otros aspectos, ejecutar las actividades de selección de la información relevantes, aplicar operaciones cognitivas, organizar la información para la elaboración e integración y, la comunicación del conocimiento. Por tanto, si los estudiantes logran integrar la información mediante la ejecución secuencial de operaciones cognitivas expuestas, habrán cumplido la primera 
condición de la comprensión conceptual; y la segunda condición, que el estudiante pueda exponer y aplicarlas.

Finalmente, en relación a los estilos de aprendizaje diferenciados entre sujetos de alto y bajo rendimiento académico universitario, Esteban, Ruiz y Cerezo (1996), señalan que existen variaciones por especialidades, como consecuencia de intervención de la variable eficacia del aprendizaje, formado por la organización, el pensamiento y la retención de datos junto con los indicadores motivacionales, que inciden en "la diferenciación significativa entre buenos y malos estudiantes de las ciencias sociales y ciencias naturales" (Esteban et al., 1996, p.164).

Marco de fundamentación de la estimulación psicofisiológica y su relación con el aprendizaje

En el segundo grupo de enfoques, se encuentra la teoría psicofisiológica del aprendizaje y su relación con los procesos cognitivos, motrices y perceptivos; donde la asimilación de conocimientos está basada en la estimulación psíquica; un fenómeno estructural donde ocurren condicionamientos del poder despertante de los estímulos al inhibirse otros impulsos sensoriales (Barrett, Barman y Scott, 2010), que vienen a ser el resultado de cambios bioquímicos formados en engramas neuronales (metacircuitos cerebrales que reposan en una constelación neuronal), asociados con el incremento de síntesis de ácidos ribonucleícos (Barrett et al., 2010; Guyton y Hall, 2005).

Específicamente la retención de la información a largo plazo donde se almacenan los conocimientos, las imágenes, los conceptos y las estrategias de actuación, son la consecuencia de reforzamientos permanentes de la sinapsis, gracias a la activación de ciertos genes y a la síntesis de proteínas (Harrison y Tong, 2009). Fundamentadas como el resultado de mecanismos neuroquímicos ocurridos en los receptores de la superficie de las membranas externas de las neuronas pos sinápticas hipocampales, donde actúa el neurotransmisor $N$-metil-Daspartato (Cárdenas y Lamprea, 2005).

Concerniente a la memoria de corto plazo, evaluadas por resonancia magnética neuronal funcional, que analiza a través de flujos sanguíneos en el cerebro mediante voxels (unidades tridimensionales dispuestas en cuadrículas de un milímetro cúbico); permiten identificar la orientación y los colores específicos de los objetos percibidos después de diez segundos de exposición a los participantes. Harrison y Tong (2009), concluyeron que la activación de 
la corteza visual anterior del cerebro está implicada tanto en la percepción de estímulos como en el almacenamiento de recuerdos a corto plazo; ayudándonos a mantener detalles visuales en función de la relevancia y del tipo de información almacenada (la orientación de objetos aparece como un patrón de actividad cerebral distinto al patrón de información del color); que utilizando algoritmos de aprendizaje automático, se pueden examinar los patrones relacionados con el recuerdo de diferentes estímulos; esto, porque los incrementos en el flujo sanguíneo del cerebro están representados por diversos vectores de la cuadrícula correspondiente a cada neurona.

En otro aspecto, McElroy y Mosteller (2006), sobre la valoración entre el recuerdo de clases, las notas y horas óptimas por la mañana o por la tarde/noche; en estudiantes universitarios, concluyen que para las clases muy difíciles, la hora óptima del día es un factor determinante en las notas de los alumnos y tiene efecto en el grado de recuerdo de las clases del semestre anterior, además de las implicaciones ovias, tanto en estudiantes como en los profesores y trabajadores administrativos. Este hecho tiene concordancia con la regulación del marcador circadiano (ubicado en el núcleo supraquiasmático), donde el mayor rendimiento en actividades cognitivas, como la memoria a corto plazo, ocurren en horarios de madrugada (Hofstra y Weerd, 2008).

Por su parte, Fawcett y Nicolson (2004), Malm y colegas (1998), sobre la intervención del cerebelo en el proceso de aprendizaje, señalan que está implicado en la automatización de cualquier habilidad sea motriz o cognitiva; por ello los pacientes con daño cerebelar presentan déficits en atención y memoria de trabajo. Algunos resultados más notables fueron la mala ejecución de los cuatro grupos con dislexia en tareas de estabilidad de postura y sacudida de miembros; vinculándose a ello los resultados mediante estudios con tomografía por emisión de positrones al $80 \%$ de niños con señales de anormalidad cerebelar. También, Thach (1996), indica que la cadena causal ontogenética del daño cerebelar, comienza con problemas de equilibrio, de habilidades motrices y de escritura, llevando menor fluidez y velocidad en la lectura. Fundamentalmente, el cerebelo está relacionado con el habla interno, una de las claves de la lectura fluida, que viene a ser la capacidad de articular a nivel subvocal. 
Estudios relacionados con la estimulación psíquica y el proceso de enseñanza aprendizaje en la asimilación de contenidos, la orientación de la prevención y la autoeficacia

En primer lugar, se pone de manifiesto la relación existente entre la disposición, el incremento de la capacidad de aprendizaje en estudiantes universitarios y, la orientación del modelado cognitivo en la resolución de problemas de prevención; revelando Sue-Chan y Wood (2009), que los temas más sensibles en la resolución de problemas, tras un programa de formación con un mes de seguimiento y evaluadas independientemente por dos jueces, fueron las vinculadas a la promoción y orientación de la prevención de la salud pública. La necesidad de comprender el pensamiento de los educandos y la suficiente disponibilidad de recursos didácticos, evitar advertencias frecuentes ni exagerar la importancia del modelado perfecto, porque afectan los resultados en aquellos educandos aprensivos de los programas preventivos.

Examinando el papel mediador de las estrategias psicofísicas (serie de actividades y movimientos preparatorios) en la enseñanza aprendizaje y la autoeficacia en 194 estudiantes norteamericanos, Gao y Newton (2009), concluyen que este tipo de estrategias ejercen diversas habilidades, primero, porque contribuye a la persistencia y el esfuerzo de los sujetos, quiénes además consideran de utilidad para la salud física; y segundo, porque ayuda hacer mejor las cosas fuera de la clase y permite manejar mejor la ansiedad. Los autores consideran que la enseñanza y la autoeficacia, fueron los predictores más importantes en los ámbitos académicos de la estrategia. Los alumnos con alta capacidad de creencias de autoeficicacia, son más propensos también a usar estrategias eficaces para mejorar la adquisición y retención de la información, que aquellos con menores niveles de autoeficacia.

Por su parte, Gheorghiu y Molz (2008), estudiaron a 177 sujetos (10-21 años de edad, Universidad de Giessen, Alemania) en grupos de 8 a10 estudiantes en sesiones de 40 a 50 minutos para medir la relajación y sugestión. Se aplicaron la relajación y flexión y, masajes en los músculos del cuello en posición sentado sobre una silla con la espalda erguida (con libre movimiento de la cabeza). Concluyéndose que las técnicas aplicadas conducen a un mejor rendimiento en la memoria y en otras áreas. Asimismo, los efectos evaluados son compatibles a los ejercicios cortos que causan relajación física y mental; sin embargo, dichos efectos no deberían generalizarse a priori debido a diferencias individuales en la capacidad de relajarse. 
Igualmente, las investigaciones realizadas en grupo de ratas en ocho semanas de entrenamiento en natación durante dos horas diarias (Hungría), sugieren que dichos ejercicios practicados con regularidad mejoran la memoria, incrementándose el factor neutrópico cerebral derivado y la disminución de daños oxidativos (Radak et al., 2006). Además, Manjunath y Telles (2004); Gheorghiu y Molz (2008), encontraron que la práctica de yoga y otras actividades como las posturas físicas variadas, la meditación y la relajación guiada, en un grupo de estudiantes hindúes (11 a 16 años), después de diez días de entrenamiento mejoraron hasta el $43 \%$ de rendimiento en la memoria espacial y memoria verbal. Por ello, cuando la mente y el cuerpo están relajados se incrementan la evocación de imágenes mentales, a partir de un material construido o una realidad ya conocida, esto, porque puede desprenderse de todos los bloqueos y tensiones para facilitar las capacidades de concentración de todos los procesos internos y poder liberar la imaginación (De Prado, 1991; Franco, 2008).

Recientemente, Davoli y Abrams (2009), confirmando el papel de la postura y la formación de imágenes mentales, sostienen que la visualización de una postura condiciona las respuestas del cuerpo tanto así como una postura física realmente adoptada. De allí que, en la motivación deportiva, se utilizan la visualización mental para ayudar a mecanizar los gestos y reforzar aptitudes que mejoran las destrezas deportivas. Resultando la imaginación, como la capacidad extraordinaria en la percepción del espacio peripersonal (burbuja invisible alrededor del cuerpo que se extiende varios centímetros a partir de la piel y en todas las direcciones y al espacio de la imaginación), con posibles ventajas de determinar anticipadamente si una acción es o no realista.

En segunda instancia, extendiendo la capacidad del cerebro para la adquisición y retención de información, Ramírez, Arenas y Henao (2005), distinguen el aprendizaje y la memoria, por la forma conveniente de organizar nuestros conocimientos sobre los procesos psicofisiológicos de adquisición de información. Por ejemplo, los resultados en la memoria visual muestran diferencias según el rendimiento del grupo combinado e inatento con respecto al grupo control. Esto es congruente con las conclusiones dadas por Galindo y colegas (2001), quienes advierten, especialmente en población con dificultades de atención, los defectos en el procesamiento de información visual y déficit en la percepción espacial que interfieren en la memoria visuoespacial inmediata. Por tanto, las diferencias en el rendimiento no estarían dadas a nivel de la función de almacenamiento, sino en la capacidad de recuperación de la información. Confirmándose que las dificultades de memoria visual se relacionan con deficien- 
cias en el uso de estrategias de planeación y organización para la evocación de la información almacenada, propiciando que el almacenamiento de información en memoria inmediata, sea de corta duración y con mayores dificultades en memoria a largo plazo.

Así como, Ruff, Kristjánsson y Driver (2007) utilizando resonancia magnética neuronal funcional a través de cinco ensayos pre y posprueba, evaluando el nivel del flujo sanguíneo y nivel de oxigenación, demostraron que tanto la memoria icónica soporta estímulos relacionados a la actividad en la corteza occipital, también es leída por los procesos de control en las regiones parieto frontal implicado en la atención selectiva espacial. Este sorprendente hallazgo refleja la naturaleza prolongada y altamente recursiva del procesamiento cortical. La estimulación de la corteza visual no se limita a informar, sino más bien, procesa en múltiples bucles de realimentación en paralelo.

Un determinado estímulo visual, puede persistir durante varios cientos de milisegundos. Los resultados implican al menos algunos procesos de la memoria icónica, que está relacionado con el procesamiento cortical visual como la corteza occipital lateral. Los procesos que son modulados por la actividad de la corteza visual mejoran la percepción de estímulos objetivo en relación con los distractores. La lectura de la memoria icónica puede incluir el control de procesos similares a las que subyacen la atención selectiva. Mientras que la circunvolución frontal derecha, resultó estar más activa durante posprueba que preprueba.

También, Gläscher y colegas (2009), utilizando simultáneamente las imágenes de resonancia magnética y tomografía axial computarizada, con transferencia a un marco de referencia común y, mediante técnica de mapeo de síntomas de lesión basados en voxels; estudiaron la relación entre las puntuaciones del test de inteligencia WAIS y las localizaciones de lesiones, demostrando que la corteza frontal izquierda estaba asociada con bajas puntuaciones en el índice de comprensión verbal y, la corteza frontal izquierda y parietal (localizada detrás del lóbulo frontal), asociadas también con bajas puntuaciones en el índice de memoria de trabajo, como las lesiones en la corteza parietal derecha relacionadas con bajo rendimiento en el índice de organización perceptiva.

De este modo, en la estrategia de estimulación psicofisiológica, aplicada con el objeto de ensayar, el proceso de generación del enfoque de la atención con énfasis en concentración isométrica así como la reconstrucción de contenidos en diversos tópicos y disciplinas. Abregú (2007), señala que los resultados más importantes son el bloqueo significativo de 
estímulos distractores de ruido, de postura y de compañía a una proporción promedio de $p=.74$, en la formación de imágenes mentales $(p=.79)$, la relajación moderada hasta en 77,9\% de estudiantes universitarios $(\mathrm{n}=172)$ y la reconstrucción promedio de contenidos en $P=0.69$ $(D E=0.14)$. Los resultados están fundamentados en que, la estrategia vivencial está basada en la estimulación simultánea del sistema nervioso y la relajación de los participantes.

En consecuencia, se recomienda establecer acciones psicopedagógicas orientadas a potenciar los procesos implicados con las habilidades para la planeación y organización de la información, organizar la estimulación de la memoria icónica, de trabajo y memoria visual, y, trabajar sobre la base de constructos teóricos vinculados con las técnicas y estrategias didácticas activas.

Partiendo de lo expuesto y aplicando los modelos teóricos descritos, el diseño de la estrategia didáctica de estimulación vivencial (EDEV) para mejorar la asimilación de contenidos en S-T, está fundamentado en la estimulación simultánea del sistema nervioso a nivel de hemisferios cerebrales, del fortalecimiento del tallo cerebral, el neocórtex, el sistema límbico y el cerebelo. Además, de la formación de experiencias cognitivas y afectivas, la práctica física y relajación; concordantes al modelo psicofisiológico del aprendizaje cerebral de anclajes y reencuadres, orientados a facilitar la fijación de contenidos mediante la estimulación psíquica, y la solución consistente en diferentes escenarios (Carpio, 1996). Promovíéndose, de esta manera, las competencias en la construcción del conocimiento y el auto aprendizaje.

Tras la revisión realizada, el objetivo del trabajo, es diseñar y aplicar la EDEV comparando en cinco grupos de estudiantes universitarios (cuatro de intervención y uno de referencia), pertenecientes a la Universidad Nacional Agraria de la Selva (Perú), para evaluar la asimilación de contenidos y la reconstrucción de rasgos icónicos sobre la S-T. Siendo el objetivo subsecuente, socializar las técnicas de estimulación psíquica como hábito en la asimilación de conocimientos y por consiguiente, mejorar los rendimientos académicos y laborales y, comprobar experimentalmente los cambios en la práctica actitudinal de los participantes.

A partir de los objetivos mencionados se plantean la siguiente hipótesis:

Aquellos estudiantes que experimentaron la EDEV, mostrarán un incremento significativo en la asimilación de contenidos y en la reconstrucción de rasgos icónicos sobre la salud en el trabajo, en comparación con aquellos estudiantes que no participaron en la aplicación. 


\section{Método}

\section{Participantes}

En el estudio se incluyeron a 125 sujetos distribuidos equitativamente en cinco grupos naturales en aula, que asistieron el año académico 2008 a la licenciatura de administración de empresas y al de ingeniería en industrias alimentarias de la Universidad Nacional Agraria de la Selva (UNAS) Perú. Los grupos, uno al cuatro participaron independientemente en lacondición de intervención y el quinto grupo actuó de referencia. Tanto los del grupo de intervención como el de referencia estuvieron conformados, cada uno, por 25 sujetos; de los cuales $49 \%$ eran varones y $51 \%$ mujeres, con edades entre los 18 y 20 años (media=19.48 años, DT= $.51)$.

\section{Instrumentos}

Para la evaluación de la variable asimilación de contenidos sobre S-T, se utilizaron un test de rendimiento académico sobre la salud en el trabajo (ARIST), diseñado para administrar colectivamente a sujetos del nivel universitario de 17 años de edad en adelante.

La composición de factores y los ítems del ARIST fueron evaluados por dos expertos en salud ocupacional, cuyas opiniones según el coeficiente de concordancia de Spearman, resultante entre ambos, fue apropiada $\left(r_{s}=.801, p<.01\right)$. En su versión original constaba de 155 ítems, agrupados en ocho factores, quedando establecido la siguiente composición: ambiente de trabajo (20 ítems), estrés y burnout (30 ítems), ergonomía: exposición a medios físicos (20 ítems), ergonomía: exposición a medios químicos (20 ítems), fatiga física y mental (15 ítems), clima laboral (20 ítems), riesgos psicosociales (20 ítems), seguridad y salud en uso de agroquímicos (10 ítems).

Los factores de estudio, como la exposición química, fueron revisados en diversos países por Espinoza y colegas (2005) y, sobre la carga física laboral en Gutierrez, Martínez y Rueda (2004).

Para evaluar la validez y confiabilidad del instrumento, se aplicó en una muestra piloto de estudiantes universitarios. Procediéndose a probar la validez conceptual o teórica del 
instrumento (construct validity), llevando a cabo un análisis factorial exploratorio (método de componentes principales) con rotación varimax (normalización KMO), partiendo de un criterio doble: únicamente se tomaron en cuenta las preguntas que 1) alcanzaron pesos factoriales iguales o superiores a 0,50 y 2) figuraron en un solo factor. Este procedimiento de extracción permitió identificar la manera en que las variables de interés se agrupan en uno o más factores, que se presume comparten determinadas premisas teóricas y conceptuales. En la tabla 1. se resumen los resultados del análisis factorial exploratorio. Se formaron tres factores, cada uno con valores propios superiores a 1.0 que en conjunto explican $64.10 \%$ de la varianza total. La medida de adecuación de la muestra de Kaiser-Meyer-Olkin fue de .574, mientras que la prueba de esfericidad de Barlett dio un resultado significativo $\left(\mathrm{X}^{2}=236.736, \mathrm{gl}=28\right.$, $\mathrm{p}=.000)$.

Tabla 1. Análisis factorial exploratorio: prueba de validez conceptual del ARIST por método de componentes principales y rotación varimax (normalización de Kaiser) y valores del coeficiente alfa de Cronbach

\begin{tabular}{|c|c|c|c|c|c|c|c|c|}
\hline \multirow[t]{2}{*}{ Factores y conglomerados } & \multirow{2}{*}{$\begin{array}{c}\mathrm{N}^{0} \\
\text { de } \\
\text { Ítems } \\
\end{array}$} & \multicolumn{3}{|c|}{$\begin{array}{l}\text { Pesos factoriales } \\
\text { por conglomerado }\end{array}$} & \multirow[t]{2}{*}{ Autovalores } & $\%$ & $\%$ & $\begin{array}{c}\text { Alfa } \\
\text { de }\end{array}$ \\
\hline & & 1 & 2 & 3 & & \multicolumn{3}{|c|}{ de varianza acumulado Cronbach } \\
\hline F1: Ambiente de trabajo y ergonomía & & & & & 2.421 & 30.258 & 30.258 & \\
\hline 1.1: Ambiente de trabajo & 15 & 0.943 & & & & & & 0.835 \\
\hline 1.2: Exposición a medios físicos & 12 & 0.931 & & & & & & 0.801 \\
\hline 1.3: Exposición a medios químicos & 12 & 0.721 & & & & & & 0,801 \\
\hline F2: Estrés y burnout y, riesgo psicosocial & & & & & 1.588 & 19.85 & 50.108 & \\
\hline 2.1: Riesgos de origen psicosocial & 8 & & 0.789 & & & & & 0,599 \\
\hline 2.2: Estrés y burnout & 10 & & 0.606 & & & & & 0,620 \\
\hline 2.3: Fatiga física y mental & 5 & & 0.548 & & & & & 0,532 \\
\hline F3: Clima laboral y salud uso de agroquímicos & & & & & 1.119 & 13.992 & 64.100 & \\
\hline 3.1: Clima laboral & 15 & & & 0.804 & & & & 0,825 \\
\hline 3.2: Seguridad-salud en uso de agroquímicos & 3 & & & 0.689 & & & & 0,669 \\
\hline
\end{tabular}

ARIST= Test de rendimiento académico y reconstrucción de rasgos icónicos sobre salud en el trabajo.

Posteriormente, se realizó un análisis de confiabilidad, para lo cual se calculó el coeficiente alfa de Cronbach, considerando las variables en su conjunto y, adicionalmente, tal y como quedaron agrupadas en cada uno de los factores. Para la coherencia interna se aceptó un coeficiente de correlación alfa entre .532 y .835. Por lo que hace a la confiabilidad, el alfa de Cronbach global del instrumento de .824; cabe mencionar que con este procedimiento se eli- 
minó 75 preguntas, quedando la versión definitiva del instrumento compuesto por 80 preguntas, con cuatro alternativas de respuesta cada uno, divididos en cinco factores, cuya valoración es un punto por cada acierto (eneatipos $7-8-9=62$ a 80 puntos; eneatipos $4-5-6=20$ a 61puntos; eneatipos 1-2-3=1 a 10 puntos), resultando el punto de corte 19 puntos para un rendimiento inferior.

La confiablidad del ARIST también fue determinada complementariamente a partir de las puntuaciones obtenidas, resultando adecuadas según el coeficiente de confiabilidad de Küder-Richardson: K-R21=.92 (según Gronlund, 1974, p. 126-129; para pruebas de rendimiento académico son apropiadas a partir de K-R21=.61), confirmándose además, la utilidad del test, según el cálculo del error estándar de medida, cuyo resultado es: $E S m=3.72$ (también según Gronlund, 1994, p. 22-23), es un indicador apropiado para pruebas de 48 a 89 ítems, siendo el límite máximo $E S m=4.00)$. Asimismo, la validez de constructo del ARIST realizada a posteriori, aplicación de la estrategia didáctica vivencial, está circunscrita en la proposición confirmatoria siguiente: el incremento en las puntuaciones de asimilación de conocimientos sobre la salud en el trabajo, tiene relación lineal directa y positiva con la proporción de rasgos icónicos resueltos por el estudiante (Fig. 1).

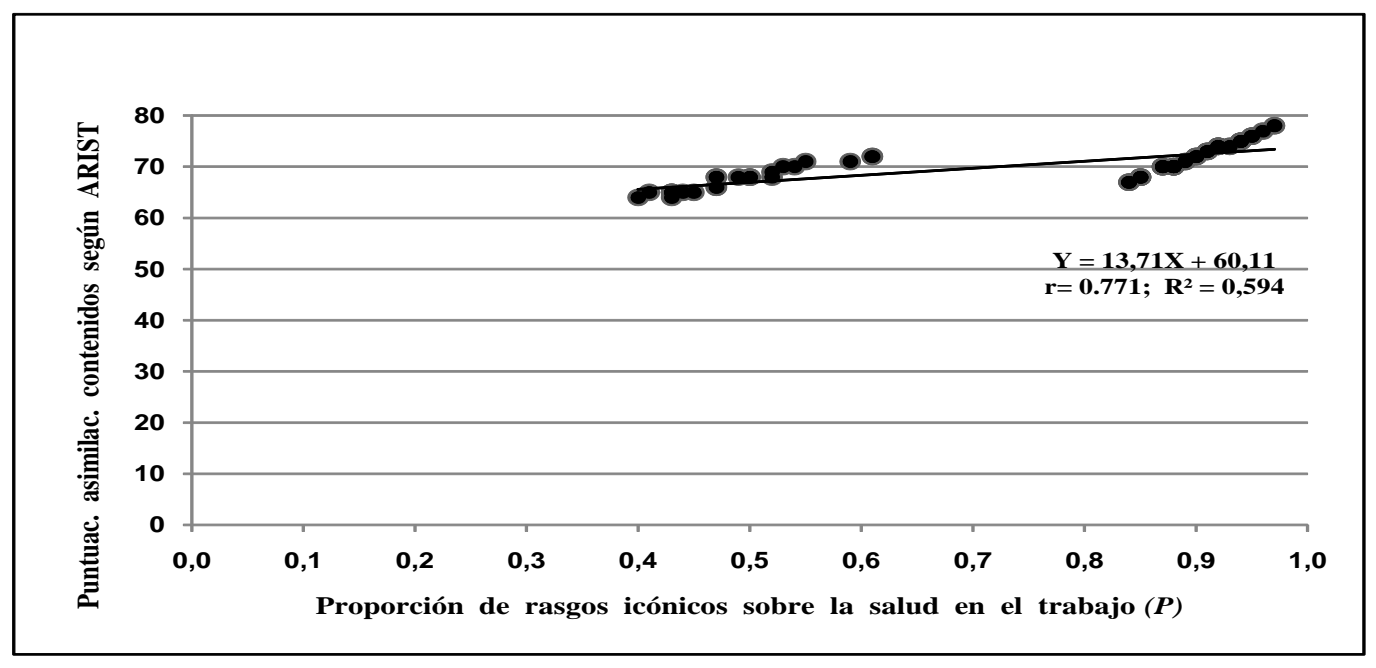

N= 75 (niveles de intervención: A, B y C)

** altamente significativo al nivel $p<.01$

ARIST=Test de rdmto. académico y reconstrucción de rasgos icónicos sobre la S-T

Figura 1 . Correlación entre puntuaciones de asimilación de contenidos y proporción promedio $(\mathrm{P})$ en la reconstrucción de rasgos icónicos sobre salud en el trabajo 


\section{Procedimiento}

Un mes antes de aplicar la EDEV se entrenó a la población de estudiantes, durante cuatro semanas en la aplicación de las técnicas gráficas de análisis, comprensión y resumen de contenidos, que incluye al mapa mental. Luego se procedió inmediatamente a recoger y evaluar los materiales construídos en mapa mental sobre la S-T. Para potenciar la comunicación didáctica, preparar las condiciones y ensayar la secuencia de aplicación de cada una de las técnicas conformantes de la estrategia didáctica de estimulación; y evaluar las condiciones ambientales en aula, se capacitó en conjunto mediante modelamiento conductual, dos semanas antes de la intervención experimental y por espacio de una semana, en las técnicas de autorrelajación digitoterapeútica $(\mathrm{AD})$, retroalimentación visual $(\mathrm{RV})$ y concentración isométrica (CIS).

Las características de las técnicas empleadas en la EDEV son:

1. Técnica de mapa mental (MM) (técnica gráfica de Novak y Gowin, 1988), empleado en la construcción de contenidos sobre S-T, porque aumentan el interés en el aprendizaje, ayudan a jerarquizar los conceptos relacionando con los conocimientos previos; favorecen el análisis y comprensión, la identificación de variables y sus relaciones causales (Boggino, 2002; Buzan y Dottino, 2005; Ledo, Vidal y Vialart, 2007).

2. Técnica de $A D$, que consiste en dar una ligera presión digital en la nuca y costados del cuello (altura de músculos esplenio y trapecio) usando tres dedos del medio de ambas manos, con los ojos cerrados y sin límite de tiempo, dando giros desde afuera hacia adentro y la inmediata flexión progresiva de brazos hacia atrás y levantando simultáneamente la cabeza hacia atrás. Técnica adaptada por su efecto psicofisiológico y cognitivo (Fig. 2) y utilizada masivamente en tratamientos de ansiedad (Cautela y Groden, 1985), por sus efectos paralelos de relajación emocional, que conducen automáticamente a la relajación del sistema nervioso central y autónomo.

3. Técnica de $R V$, que consiste en observar globalmente el material construido durante 3,5 min como máximo, pasado este tiempo se presentarían cansancio mental y su consecuente desviación de la atención (Reinoso, 2001). 
4. Técnica de CIS, son movimientos estáticos con ojos cerrados, concentración en la imagen del material gráfico construido (ejecutado sólo después de aplicar las técnicas de AD y de RV), que consiste en la combinación secuencial y de cambios autónomos (menor a un minuto), entre la flexión profunda de rodillas con brazos paralelos al frente (posturas “1”, "3”, 5"), y la flexión profunda de rodillas con brazos tocando las rodillas y espalda erguidas, posturas “2” y “4” (Fig. 3). Técnica diseñada considerando que la estimulación de músculos y el equilibrio presentan ritmos de menor fatiga, contribuyen al despertar kinestésico y favorecen la formación de imágenes del material construido (Abregú, 2007).

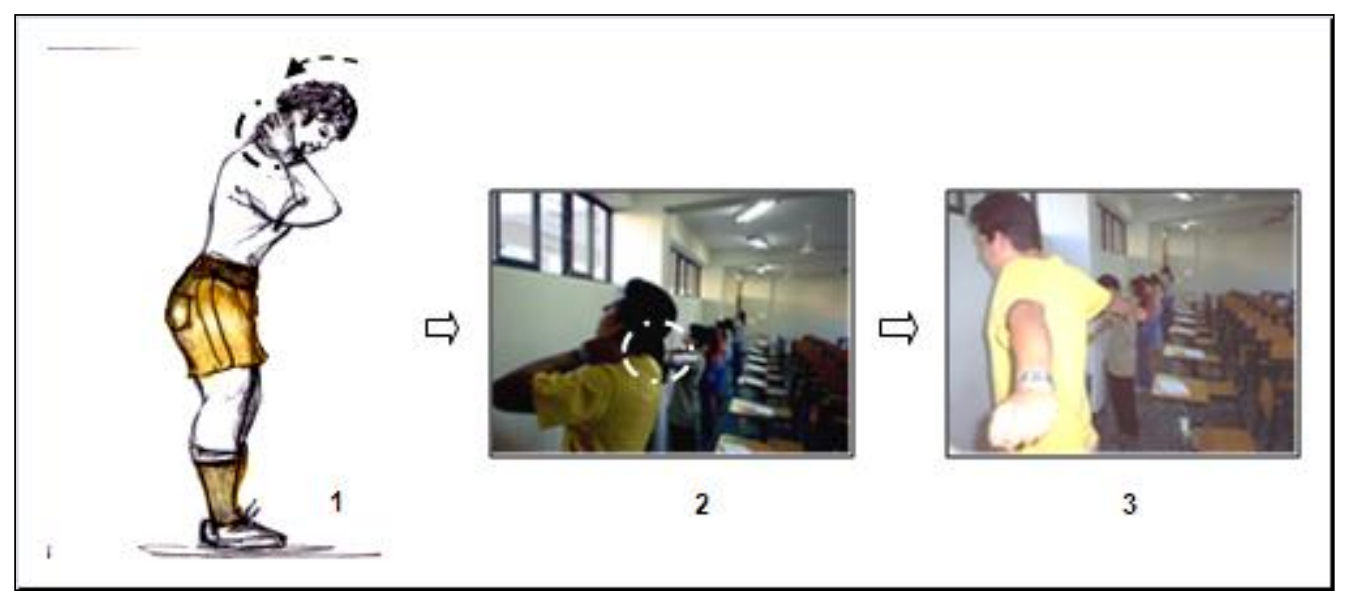

Figura 2. Posturas en la técnica de autorrelajación digitoterapéutica

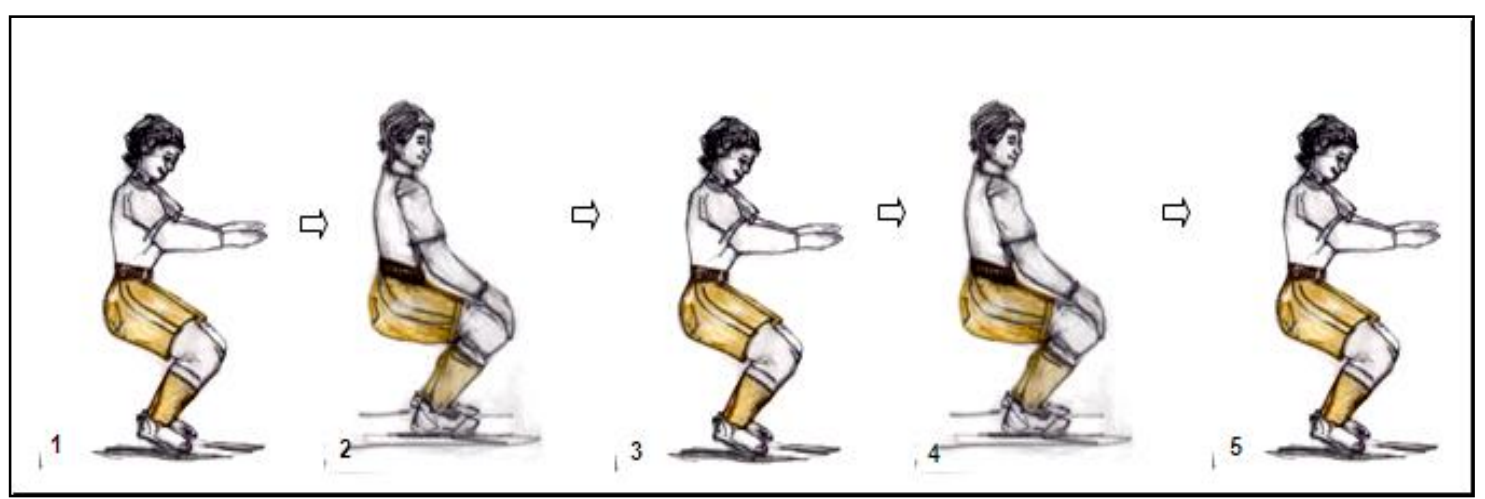

Figura 3. Posturas en la técnica de concentración isométrica

La asignación de sujetos en cada semestre y especialidad fue al azar, aplicándose para ello el criterio de doble ciego. Primero, los estudiantes desconocían sobre el tratamiento del estudio y segundo, desconocían sobre la pertenencia al grupo de intervención o al grupo con- 
trol. Con ese objetivo se establecieron cuatro niveles de tratamiento de la variable independiente (A, B, C, D).

La secuencia para aplicar el diseño experimental empleando la EDEV fue: XYABABA ( $\mathrm{X}=$ téc. $\mathrm{AD}, \mathrm{Y}=$ téc. $\mathrm{RV}$; Téc. CIS: A-B-A-B-A, siendo A=posturas 1, 3, 5; $\mathrm{B}=$ posturas 2, 4). Luego la reconstrucción mental inmediata de rasgos icónicos (RI) sobre la salud en el trabajo en una hoja de papel y sin límite de tiempo a cargo del estudiante (Fig. 4). La reconstrucción de RI descritas por Llorente (1999), como forma y número de figuras, símbolos lógicos, número y categorías semánticas, ubicación y forma, palabras, códigos y otros usos semióticos sobre la S-T; se cuantificaron en proporciones $(P)$, obtenidas comparando la frecuencia de RI construidos con libre amplitud en pre prueba y los RI reconstruídos sin límite de tiempo en pos prueba.

\begin{tabular}{|c|c|c|c|c|c|}
\hline \multirow[b]{2}{*}{ Técnicas de la estrategia didáctica } & \multicolumn{4}{|c|}{ Nivel de intervención } & \multirow{2}{*}{$\begin{array}{c}\text { Referencia } \\
\text { E }\end{array}$} \\
\hline & $\mathbf{A}$ & B & $\mathrm{C}$ & D & \\
\hline 1 Mapa mental (MM) & $\square$ & $\square$ & $\square$ & & \\
\hline 2 Autorrelajación digitoterapeútica (AD) & $\square$ & $\square$ & $\square$ & $\square$ & \\
\hline 3 Retroalimentación visual (RV) & $\square$ & $\square$ & $\square$ & $\square$ & \\
\hline 4 Concentración isométrica (CIS) & $\square$ & & & & \\
\hline 5 Práctica deportica habitual (PD) & $\square$ & $\square$ & & & \\
\hline \multicolumn{6}{|l|}{ Variables de evaluación: } \\
\hline 1 Asimilación de contenidos sobre S-T & $\square$ & $\square$ & $\square$ & $\square$ & $\square$ \\
\hline 2 Reconstrucción de rasgos icónicos sobre S-T & $\square$ & $\square$ & $\square$ & & \\
\hline
\end{tabular}

Figura 4. Composición de técnicas de la estrategia didáctica de estimulación vivencial (EDEV) y, variables de evaluación según niveles de intervención

Inmediatamente después, se procedió a evaluar la variable asimilación de contenidos sobre S-T mediante el test de rendimiento académico (ARIST), tanto en los grupos de intervención como en el de control.

Durante la experimentación, se ubicaron a cada uno de los estudiantes entre $2,5 \mathrm{~m}$ de distancia y de 8 a 9 personas como máximo por sesión en aula. Teniendo en cuenta que el mayor rendimiento en actividades cognitivas, como la memoria a corto plazo, ocurren en horarios de madrugada (Hofstra y Weerd, 2008) y la hora óptima del día es un factor determi- 
nante en las notas de los alumnos (McElroy y Mosteller, 2006), la aplicación de la EDEV fue desde las 7.30 am hasta las 10.30 am como máximo.

\section{Diseño y Análisis Estadístico}

El tipo de estudio, según su relación con la práctica es aplicada, por su naturaleza es empírica y metodológica y, es de carácter comparativo de causa y efecto. Se aplicó el diseño experimental totalmente al azar de una variable independiente (VI), con cuatro niveles de intervención y otra de referencia. Las razones del uso están fundamentadas, en que, el diseño con más de dos condiciones o niveles de tratamiento, permiten obtener información exacta sobre la relación entre las variables, dado que podremos establecer la relación funcional entre ambas (diseños funcionales). Que pretendemos determinar la relación existente entre el número de técnicas que conforman la EDEV y la asimilación de contenidos y la reconstrucción de rasgos iconicos sobre S-T.

Con el objetivo de establecer dicha relación, se seleccionó arbitrariamente los siguientes valores de la VI: 5, 4, 3, 2 y cero (0) técnicas; siendo obvio, que a medida que se utilice más valores de la VI, estaremos en mejores condiciones para conocer la relación funcional entre ambas variables. Una vez que la relación funcional quede bien establecida, este procedimiento permite interpolar y extrapolar valores que no han sido probados experimentalmente. Asimismo, el experimento funcional posee gran importancia en la investigación, ya que no solo permite establecer con mayor precisión la relación existente entre dos variables, sino también se puede derivar un modelo cuantitativo que presente la función más exacta (Arnau, 1995, p. 65-66).

En la evaluación de efectos de la EDEV sobre la asimilación de contenidos y reconstrucción de rasgos icónicos en ST, como en el resto de las variables estudiadas, se compararon las medias entre cada nivel de tratamiento y el grupo control, a través de un análisis de varianza pos prueba, previa evaluación de homogeneidad de varianzas de Levene, calculándose la desviación estándar, el error típico, el intervalo de confianza (IC: 95\%) y la razón “ $F$ ” $(p=, 001)$; además de la transformación logarítmica de puntuaciones (por distribución heterogénea de varianzas) y, las pruebas post hoc y de subconjuntos homogéneos de Tukey. 
Considerando que la construcción de contenidos en RI tiene libre amplitud (es válido que un mismo tópico pueda construirse por ejemplo, en 25 ó $90 \mathrm{RI}$, siendo $P=1,000$ reconstrucción total y $P=, 000$ reconstrucción nula), se determinaron la proporción promedio $(P)$. Se aplicó la prueba $\mathrm{Ji}$-cuadrado, para evaluar la contingencia de casos entre los grupos de intervención y las variables relajación, formación de imagen mental y bloqueo de estímulos distractores. Para todos los procesos se utilizaron el programa informático SPSS versión 18.0 para Windows ${ }^{\circledR}$.

\section{Resultados}

Asimilación de contenidos y reconstrucción de rasgos icónicos sobre la salud en el trabajo

Las puntuaciones en la asimilación de contenidos, determinados mediante el test de rendimiento académico ARIST, como efecto de aplicar la EDEV en alumnos/as del nivel universitario y sometidos a la prueba de Kolmogorov-Smirnov (KS), presentan una distribución normal de datos (diferencias absolutas son menores al coeficiente " $Z$ " de $K S$ en todo los casos, A: $K S=.801, p<.543$; en B: $K S=.723, p<.674 ; \mathrm{C}: K S=.838, p<.484 ; \mathrm{D}: K S=.731$, $p<.660$; y en GC: $K S=.889, p<.409$ ); que igualmente contrastadas mediante análisis gráfico de distribución de las puntuaciones de tendencia central y de posición (Fig. 5), permiten establecer con seguridad y confianza los resultados de las variables en estudio.

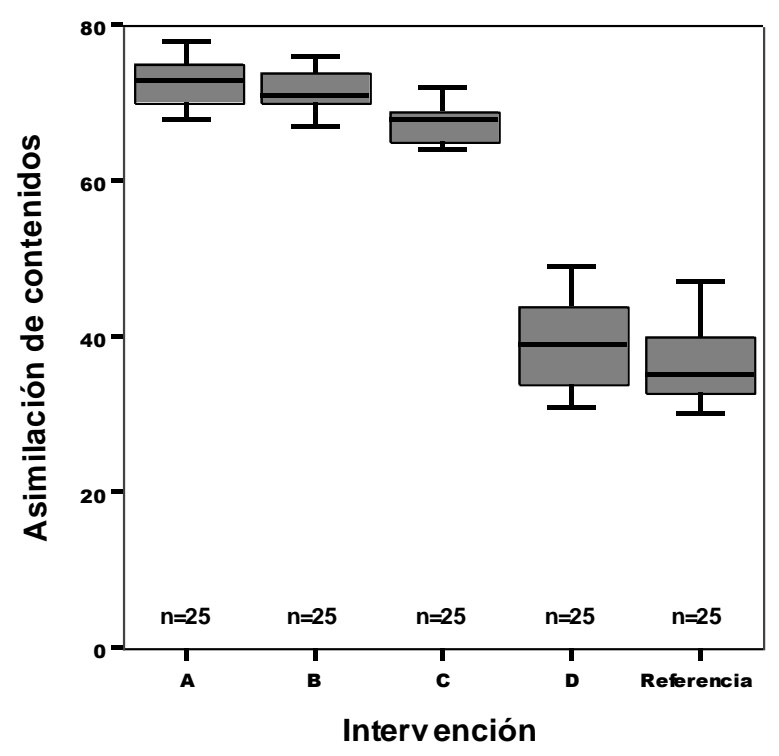

Figura 5. Distribución de puntuaciones de asimilación sobre la salud en el trabajo evaluados mediante ARIST en el grupo de intervención y de referencia 
Previo análisis de varianza de puntuaciones antes de la aplicación de la variable independiente (EDEV), la variable dependiente (VD) asimilación de contenidos sobre S-T en preprueba, muestra ausencia de diferencias significativas entre los cuatro grupos de intervención y el de control (Tabla 2). Indicando un control adecuado de variables extrañas en aula, que la selección es aleatoria y homogénea en los participantes y el desempeño también es homogéneo en la VD; asimismo, la variación del desempeño en posprueba es el efecto de la VI.

El primer resultado, nos indica que los participantes en el grupo de intervención muestran una media de rendimiento superior en la asimilación de contendios sobre S-T, tanto en el grupo "A" $(X=118.56)$ como en el grupo "B" $(X=115.96)$, que en los grupos "C" $(X=$ 109.60) y "D” (X=63.16). Además, el siguiente resultado obtenido mediante el ANOVA, señala que entre el grupo "A" y "B" no existen diferencias significativas, tampoco entre el grupo de intervención " $\mathrm{D}$ ” y el grupo de referencia.

Entre los grupos de intervención, existen dos conglomerados con rendimiento homogéneo sobre la salud en el trabajo: 1) formado por los grupos A-B y, 2) conformado por el grupo "D" y los participantes del grupo de control $(X=60.24)$. Asimismo, según pos prueba HSD de Tukey, además de la presencia de diferencias significativas entre cada uno de los dos conglomerados (A-B versus "C"), igualmente existen diferencias entre el conglomerado A-B respecto el segundo conglomerado ( $\mathrm{D}$ - grupo de referencia) (Tabla 2).

Los resultados del ANOVA de las diferencias pos prueba (Tabla 3), basados en las puntuaciones del rendimiento sobre la salud en el trabajo, son significativamente diferentes $(F=316.35 ; p<.0001)$. Realizada las comparaciones múltiples en la variable asimilación de contenidos, los participantes del grupo de tratamiento en el nivel "A" confirman un mayor rendimiento que aquellos del nivel "C", " $\mathrm{D}$ " y del grupo de referencia $(p<.0001)$; en cambio, los estudiantes del grupo "A" no muestran dichas diferencias frente a los participantes del grupo "B” $(p<.658)$.

Respecto al grupo de intervención en el nivel "C", el rendimiento sobre la S-T es peor que aquellos alumnos/as participantes en los dos niveles de tratamiento (A,B); sin embargo, no existen diferencias respecto a aquellos que intervinieron dentro del nivel " $\mathrm{D}$ " $(p<.551)$. 
Tabla 2. Promedio y DE de puntajes en asimilación de contenidos sobre salud en el trabajo evaluados en pre y posprueba mediante el ARIST como efecto de aplicación de la EDEV

\begin{tabular}{|c|c|c|c|c|c|c|c|c|}
\hline $\begin{array}{c}\text { Grupo } \\
\text { Nivel } \\
\end{array}$ & $\mathbf{N}$ & $\begin{array}{r}G \\
\text { Varon } \\
\end{array}$ & $\begin{array}{l}\text { ero } \\
\text { Mujeres } \\
\end{array}$ & $\begin{array}{l}\text { Media } \\
\text { pre test }\end{array}$ & \multirow[t]{2}{*}{$\begin{array}{c}D E \\
\text { pretest }\end{array}$} & \multirow[t]{2}{*}{$\begin{array}{c}\text { Media } \\
\text { pos test }\end{array}$} & \multirow[t]{2}{*}{$\begin{array}{c}D E \\
\text { pos test }\end{array}$} & \multirow[t]{2}{*}{$I C(95 \%)$} \\
\hline \multicolumn{5}{|c|}{ De intervención } & & & & \\
\hline A & 25 & 13 & 12 & 40.89 & 4.10 & 73.00 & 2.84 & $71.83-74.17$ \\
\hline B & 25 & 13 & 12 & 39.87 & 4.44 & 71.32 & 2.87 & $70.14-72.50$ \\
\hline $\mathrm{C}$ & 25 & 12 & 13 & 42.41 & 4.98 & 67.44 & 2.53 & $66.39-68.49$ \\
\hline D & 25 & 13 & 12 & 40.38 & 5.05 & 38.84 & 6.62 & $36.11-41.57$ \\
\hline De referenci & & & & & & & & \\
\hline E & 25 & 13 & 12 & 41.36 & 5.29 & 37.08 & 4.92 & 35.05 - 39.11 \\
\hline
\end{tabular}

$D E=$ Desviación estandar; $\mathrm{IC}=$ Intervalo de confianza para la media.

ARIST $=$ Test de rendimiento académico y reconstrucción de rasgos icónicos sobre salud en el trabajo.

$\mathrm{EDEV}=$ Estrategia didáctica de estimulación vivencial; $\mathrm{PD}=$ práctica deportiva; $\mathrm{CIS}=$ concentración isométrica.

A: EDEV; B: (EDEV-CIS); C: (MM+AD+RV); D: AD+RV.

$\mathrm{AD}=$ Autorelajación digitoterapéutica; $\mathrm{RV}=$ Retroalimentación visual; $\mathrm{MM}=$ mapa mental.

Tabla 3. Análisis de varianza de las variables asimilación y reconstrucción de rasgos icónicos sobre salud en el trabajo.

\begin{tabular}{|c|c|c|c|c|c|c|}
\hline \multirow[t]{2}{*}{ Fuente } & \multirow[t]{2}{*}{ Gl } & \multicolumn{2}{|c|}{$\begin{array}{l}\text { Asimilación de } \\
\text { contenidos }\end{array}$} & \multirow[t]{2}{*}{ Gl } & \multicolumn{2}{|c|}{$\begin{array}{l}\text { Reconstrucción de } \\
\text { rasgos icónicos }\end{array}$} \\
\hline & & $\begin{array}{r}\text { Media } \\
\text { cuadrática }\end{array}$ & $\boldsymbol{F}$ & & $\begin{array}{l}\text { Media } \\
\text { drática }\end{array}$ & $F$ \\
\hline Inter grupos & 4 & 2.998 & $316.35 * *$ & 2 & 1.434 & $710.58 * *$ \\
\hline Intragrupos & 120 & 0.009 & & 72 & 0.002 & \\
\hline Total & 124 & & & 74 & & \\
\hline
\end{tabular}

** p<.001; $\quad \mathrm{a}=$ Transformado a logaritmo; $p=$ Proporción promedio entre construcción y reconstrucción.

El rendimiento sobre la salud en el trabajo, comparando específicamente entre los niveles de intervención con la práctica deportica $(A, B)$ y sín ella (C,D), no existen diferencias significativas $(p<.251)$. Sin embargo, el factor modulador de cambios, viene a ser la práctica de la técnica de concentración con postura isométrica (CIS) aplicado únicamente en el nivel "A", y el uso de la técnica de mapa mental empleado en los tres niveles (A,B,C). En cambio, comparando el rendimiento de los participantes del nivel " $\mathrm{B}$ " con los del nivel "C" tienen resultados similares. 
En relación al desempeño en la reconstrucción de rasgos icónicos sobre S-T, evaluados mediante ANOVA en los tres niveles que emplearon la técnica de mapa mental (A,B,C), indica la existencia de diferencias significativas $(F=710.58 ; p<.0001)$; de los cuales, aquellos que aplicaron simultáneamente la técnica CIS y la práctica deportiva en "A" y "B" son mejores respecto al nivel "C", quiénes usaron la auto relajación dígitoterapéutica y la retroalimentación visual $(p<.0001)$.

\section{Discusión y conclusiones}

Los resultados obtenidos en el estudio permiten afirmar que la aplicación de la estrategia didáctica de estimulación vivencial, produce un incremento significativo en la asimilación de contenidos y en la reconstrucción de rasgos icónicos sobre la salud en el trabajo (S-T), que comprende los hechos, conceptos y procedimientos en los tópicos de: ambiente de trabajo, exposición a medios físicos y a medios químicos, riesgos de origen psicosocial, estrés y burnout, fatiga física y mental, clima laboral y, seguridad y salud en el uso de agroquímicos; confirmándose la hipótesis del estudio. Igualmente, del cambio logrado en la formación de imagen mental a partir de un material construido y de la capacidad de bloquear diferentes estímulos distractores especialmente durante la concentración isométrica, en conjunto, pueden desprenderse que están reflejando la formación del enfoque de la atención.

Comparando los resultados del rendimiento en memoria espacial y verbal (hasta el 43\%) publicados por Manjunat y Telles (2004), como efecto de la práctica de yoga y de otras artes (10 días, edad:11-16 años), con los rendimientos por aplicación de la EDEV, difieren significativamente en la asimilación de contenidos alcanzados en los niveles "A" (5 técnicas), "B" (4 técnicas) y "C" (3 técnicas) versus grupo de referencia, cuyas variaciones porcentuales (VP) correspondientes son: $\mathrm{VP}=96.8 \%, \mathrm{VP}=92.5 \%$ y $\mathrm{VP}=81.0 \%$. Sin embargo, en el nivel "D" (2 técnicas) la variación es apenas $\mathrm{VP}=4.8 \%$; y respecto a los resultados del grupo control es inferior al de la práctica de yoga y otros ( $\mathrm{VP}=-88.8 \%$ ). Explicándose que, dos sesiones de entrenamiento y una de aplicación, únicamente en las técnicas de autorrelajación digitoterapeútica y la retroalimentación visual (RV), son insuficientes para lograr el enfoque de la atención, para asociar el aprendizaje, estimular el sistema nervioso y, mucho peor, formar una experiencia vivencial. En cambio, si incorporamos sistemáticamente las técnicas de concentración isométrica, el uso de las técnicas gráficas como el mapa mental, la autorrelajación 
digitoterapeútica, la RV y la práctica deportiva cotidiana; los resultados en la asimilación y la reconstrucción de rasgos icónicos sobre $\mathrm{S}$ - T, se potencian como ocurrió en los niveles "A" y "B".

De esta forma, se evidencian la importancia de desarrollar y socializar las técnicas vivenciales de estimulación psíquica que promueven el pensamiento simbólico, como hábito de estudio y estilo de vida; lográndose un mayor compromiso entre profesores/as y estudiantes; mejorar la asimilación de contenidos, el rendimiento académico y la calidad de la educación superior, fortaleciendo las competencias para el auto aprendizaje como las habilidades para "aprender a aprender"; consecuentemente, elevar el desempeño en la práctica profesional y laboral aplicada en la S-T (Diaz, 1997; García, 2005).

Ratificándose además, que el estudiante, solamente con el resultado de su propia experiencia vivencial, modificará su comportamiento en la asimilación de competencias y la práctica laboral (Abregú, 2007; Gao y Newton, 2009; Lacasa et al., 2002); y en tanto, el interés por la estrategia de estimulación, ejerza un efecto directo y significativo por la persistencia y el esfuerzo, perdurará su práctica, aunque de antemano el sujeto deberá estar conciente que dicha estrategia le permite mejorar su desempeño académico o laboral. Permitiendo demostrar, que la adquisición de competencias son necesarias para forjar la práctica de los valores y la motivación por la S-T; impulsando de esta manera, la cultura de prevención y control de riesgos ocupacionales señalados por García (2005), Lippke y Zielgelmann (2008) y WHO (1995).

Asimismo, permiten reafirmar que los resultados obtenidos de aplicar la EDEV, están basados en la macro estimulación del sistema nervioso ( $\mathrm{SN}$ ) a nivel de los hemisferios cerebrales, del fortalecimiento del tallo cerebral, del sistema límbico, cerebelo y el neocórtex; pero especialmente de las cortezas frontal izquierda y parietal derecha, que en interacción con el cerebelo, regulan la memoria de trabajo y la organización perceptiva (De Prado, 1991; Fawcett y Nicolson, 2004; Franco, 2008; Glascher et al., 2009; Ruff et al., 2007). Además de la formación de experiencias cognitivas y afectivas, de la práctica deportiva habitual y la relajación; que están circunscritas en el modelo psicofisiológico del aprendizaje cerebral de anclajes y reencuadres, orientado a facilitar la fijación de contenidos mediante la estimulación psíquica y la solución sostenida en diferentes escenarios señalados por Carpio (1996) y Gheorghiu y Molz (2008). 
El enfoque de la atención y la asimilación de contenidos, logrado por los participantes, está reflejado en primera instancia, al bloqueo de estímulos distractores y del mejoramiento de la formación de imágenes mentales; que en la teoría, corresponden a la inhibición de impulsos sensoriales y condicionamientos del poder despertante de los estímulos (Barret et al., 2010; Davoli y Abrams, 2009; De Prado, 1991; Franco, 2008).

En segunda instancia, por la interacción del uso de la técnica MM con la estimulación del SN y la formación de experiencias señaladas, que contribuyen en la motivación para el aprendizaje y, en la formación de esquemas mentales, incluyendo a las operaciones cognitivas de la abstracción y la asimilación de tópicos. Diferenciándose marcadamente entre los estudiantes participantes y no participantes de la EDEV, debido al aprendizaje vivencial, plasmada por la motivación en la organización de la estimulación psíquica, señalados por diversos autores (Biggs, 2001; Escoriza, 2003; Galindo et al., 2001). Teniendo correspondencia con la estimulación del lenguaje no verbal y la memoria visuoespacial inmediata y de largo plazo; teóricamente explicada, como la función de ambos hemisferios cerebrales y específicamente de la corteza frontal izquierda y corteza parietal derecha (Galindo et al., 2001; Ramírez et al., 2005; Ruff et al., 2007).

Por otro lado, a partir de la auto relajación digitoterapéutica, se ha logrado disminuir la ansiedad, generando la relajación del SN central y autónomo, para incrementar el proceso mnémico con la aplicación de la retroalimentación visual, que están convalidadas en los argumentos teóricos de Cárdenas y Lamprea (2005), en que, una mejor respuesta se logra al acompañarse de estímulos agradables o aún de estímulos desagradables.

En tercera instancia, los logros obtenidos por los participantes están reflejados por la acción de la concentración con posturas isométricas, que permiten estimular el equilibrio y las asociaciones indicadas anteriormente, potenciando de este modo la formación de imágenes mentales sobre S-T, también fundamentada teóricamente por Barret y colegas (2010) y Gheorhiu y Molz (2008), en que la concentración estática, ejerce la contracción y relajación de músculos en longitud constante y los cambios bioquímicos generados son reversibles.

Según los fundamentos psicofisiológicos descritos en la aplicación de la EDEV y el rendimiento sobre la salud en el trabajo, igualmente tienen concordancia al aprendizaje cerebral de anclajes y reencuadres (Carpio, 1996), reconocido hace varios años como el aprendi- 
zaje asociativo, porque en toda las áreas cerebrales, ocurren constantemente procesos de reorganización de circuitos neuronales, como consecuencia de la experiencia y el efecto de relacionar un estímulo con otro; adicionando la capacidad de modificación del comportamiento para la asimilación de contenidos, que en este estudio es la estimulación vivencial.

Al respecto algunos autores (Barret et al., 2010; Kandell y Schwatz, 2004), han demostrado que los estímulos gráficos, como el MM y la retroalimentación visual usadas, activarían la corteza visual primaria y asociativa. En cambio, con la verbalización interna, se está estimulando en términos globales al área de Broca; con el pensamiento simbólico, al lóbulo frontal; $\mathrm{y}$, específicamente la concentración isométrica aplicada, tiene vinculación con algunos aportes de Cárdenas y Lamprea (2005), Fawcett y Nicolson (2004) y Malm y colaboradores (1998), en que, las relaciones y sistemas sensoriales de MM y retroalimentación visual y sistemas de ejecución motriz (auto relajación digitoterapeútica y concentración isométrica), activarían la memoria procedimental, generando globalmente las conexiones corticales y la sincronización subcortical en el hipocampo, el septum y el cerebelo.

\section{Agradecimientos}

Este trabajo financiado como parte del programa de formación permanente de la Fundación Carolina (España).

\section{Referencias}

Abregú, L.F. (2007). La reconstrucción de contenidos mediante la generación del enfoque de la atención basado en concentración isométrica. Revista Electrónica PsicologíaCientífica.com, 6(260), 1-17. Bogotá: Psicom Editores. [En línea]. http://www.psicologíacientifica.com/publicaciones/biblioteca/artículos/ar-260-6.htm. [2009, enero 25].

Arnau, J. (1995). Diseños experimentales en psicología y educación. México: Trillas.

Barret, K., Barman, S., Scott, H. (2010). Ganong's review of medical physiology. USA: McGraw-Hill Companies.

Biggs, J. (2001). Teaching for quality learning at university. Buckinham: Open University Press.

Boggino, N. (2002). Cómo elaborar mapas conceptuales. Aprendizaje significativo globalizado. Argentina: Homo Sapiens.

Buzan, T. y Dottino, T. (2005). Mapas mentales. España: Deusto. 
Cabellos, M.R., Canabal, C., Castillo, G., Cobos, J., Gavaldón, G., Gigante, C. y otros. (2007). Estrategias de innovación docente para favorecer el aprendizaje autónomo de los estudiantes de la Universidad de Alcalá. España: Servicio de Publicaciones Universidad de Alcalá.

Cárdenas, F. y Lamprea , M. (2005). El cerebro: Aquella inestable matriz. Revista Electrónica PsicologíaCientífica.com, 6(198), 1-16. Bogotá: Psicom Editores. [En línea]. http://www.psicologíacientifica.com/publicaciones/biblioteca/artículos/ar-198-6.htm. [2006, setiembre 4].

Cardozo, S., Andino, G.S., Beatriz, A., Esquivel, B. y Espindola, E. (2008). Effectiveness of the active methods as a teaching-learning strategy in large and heterogeneous groups. Revista Cubana de Educación Médica Superior, 22(1), 1-6.

Carpio, M.M. (1996). Anclajes y reencuadres para un aprendizaje eficaz con todo el cerebro. Revista de Investigación Instituto Pedagógico “J.M. Siso Martínez”, 4(1), 12-17.

Cautela, J. y Groden, J. (1985). Técnicas de relajación. España: Ed. Martínez Roca.

Davoli, C. y Abrams, R. (2009). Reaching out with the imagination. Psychological Science, 20(3), 293-295.

Diaz, A. (1997). Didáctica y currículo. México: Paidós.

De Prado, C. (1991). La orientación e intervención expresivo creativa. España: Universidad de Santiago de Compostela.

Escoriza, J. (2005). Enseñanza de las estrategias de comprensión del lenguaje escrito: Selección y secuenciación de objetivos y contenidos. Electronic Journal of Research in Educational Psychology, 6, 3(2), 1-32.

Escoriza, J. (2003). Evaluación del conocimiento de las estrategias de comprensión lectora. Barcelona: Universitat de Barcelona.

Espinoza, MT., Partanen, T., Piñeros, M., Chaves, J., Posso, H., Monje, P. et al. (2005). Determinación del historial de exposiciones en la epidemiología ocupacional. Revista Panamericana de Salud Pública, 18(3), 187-196.

Esteban, M., Ruiz, C. y Cerezo, F. (1996). Los estilos de aprendizaje y el rendimiento en ciencias sociales y en ciencias de la naturaleza en estudiantes de secundaria. Anales de psicología, 12(2), 153-166.

Fawcett, A. y Nicolson, A. (2004). El cerebelo: su implicación en la dislexia. Electronic Journal of Research in Educational Psychology, 2(2), 35-58. 
Franco, C. (2008). Relajación creativa, creatividad motriz y autoconcepto en una muestra de niños de educación infantil. Electronic Journal of Research in Educational Psycho$\log y, 14,6(1), 29-50$.

Galindo, G., De la Peña, F., De la Rosa, N., Robles, E., Salvador, J. y Cortes, J. (2001). Análisis neuropsicológico de las características cognitivas de un grupo de adolescentes con trastorno por déficit de atención. Salud Mental, 24(4), 50-56.

Gao, Z. y Newton, M. (2009). Examining the mediating role of strategy use on students' motivation and persistence/effort in physical education. Journal of Sport Behavior, 32(3), 278-297.

García, A.B. (2005). Estudio de los enfoques de aprendizaje en estudiantes de magisterio y psicopedagogía. Electronic Journal of Research in Educational Psychology, 6, 3(2), 109-126.

Gheorghiu, V. y Molz, G. (2008). Measurement of responses to suggestions for relaxation by means of indirect and direct tests. Contemporary Hypnosis, 25(2), 78-93.

Gläscher, J., Tranel, D., Paul, L., Rudrauf, D., Rorden, C., Hornaday, A., et al. (2009). Lesion mapping of cognitive abilities linked to intelligence. Neuron, 61(5), 681-691.

Grounlud, N.E. (1974). Elaboración de tests de aprovechamiento. México: Trillas.

Guyton, A. y Hall, J. (2005). Medical physiology. Phyladelphya USA: Elsevier Sanders.

Gutiérrez, A. M., Martínez, N. P. y Rueda, M. C. (2004). Determinación de la carga física laboral en trabajadores del área de ensamblaje en una empresa manufacturera de elementos de caucho de Bogotá, 2003-2004. Revista Facultad de Medicina, 9(2), 4855.

Harrison, S.A. y Tong, F. (2009). Decoding reveals the contents of visual working memory in early visual areas. Nature, 458(1), 632-635.

Hofstra, W.A. y Weerd, A.W. (2008). How to assess circadian rhythm in humans: A review of literature. Epilepsy y Behavior, 13(1), 438-444.

Kandel, E.R. y Schwatz, J.H. (2004). Essential of neural science and behavior. EE.UU: Ed. McGrawHill.

Lacasa, P., Mudarra, M., Del Castillo, H., García, A. y Martínez, R. (2002). Contraviolencia: Una experiencia multimedia en las aulas. Revista Electrónica Interuniversitaria de Formación del Profesorado, 5(4), 1-5.

Ledo, M.V., Vidal, N.V. y Vialart, D.R. (2007). Conceptual maps. A learning strategy. Revista Cubana de Educación Médica Superior, 21(3), 1-5. 
Lippke, S. y Ziegelmann, J.P. (2008). Theory-based health behavior change: Developing, testing, and applying theories for evidence-based interventions. Applied Psychology an International Review, 57(4), 698-716.

Llorente, E. (1999). Imágenes en la enseñanza. Revista Psicodidáctica, 9(1), 1-19.

Malm, J., Kristensen, B., Karlsson, T., Carlberg, B., Fagerlund, M., y Olsson, T. (1998). Cognitive impairment in young adults with infratentorial infarcts. Neurology, 51(1), 433440.

Manjunath, N. K. y Telles, S. (2004). Spatial and verbal memory test scores following yoga and fine arts camps for school children. Indian J Physiol Pharmacol, 48(3), 353-356.

McElroy, T. y Mosteller, L. (2006). La influencia del tipo circadiano, la hora del día y la dificultad de la clase sobre las notas de los estudiantes. Electronic Journal of Research in Educational Psychology, 10, 4(3), 611-622.

Novak, J. y Gowin B.D. (1988). Aprendiendo a aprender. España: Martínez Roca.

Radak, Z., Toldy, A., Szabo, Z., Siamilis, S., Nyakas, C., Silye, G. et al. (2006). The effects of training and detraining on memory, neurotrophins and oxidative stree markers in rat brain. Neurochemistry Internacional, 49(4), 387-392.

Ramírez, L.A., Arenas, A. y Henao, G. (2005). Caracterización de la memoria visual, semántica y auditiva en niños y niñas con déficit de atención tipo combinado, predominantemente inatento y un grupo control. Electronic Journal of Research in Educational Psychology, 7, 3(3), 89-108.

Ruff, Ch. C., Kristjánsson, Á. y Driver, J. (2007). Readout from iconic memory and selective spatial attention involve similar neural processes. Psychological Science, 18(10), 901-909.

Sánchez, B. (2007). Aplicación de estrategias de aprendizaje autónomo en la asignatura «Principios de cinesiterapia». España: Servicio de Publicaciones Universidad de Alcalá.

Sue-Chan, Ch. y Wood, R.E. (2009). The moderating effect of trainee implicit beliefs on the relationship between cognitive modeling orientation and training outcomes. Applied Psychology an International Review, 58(2), 304-335.

Thach, W.T. (1996). On the specific role of the cerebellum in motor learning and cognition: Clues from PET activation and lesion studies in man. Behavioural and Brain Sciences, 19(1), 411-431.

World Health Organization (WHO). (1995). Global strategy on occupational health for all: the way to health at work. Panamerican Journal of Public Health, 119(5), 442-450. 